\title{
Acuerdo UE-MERCOSUR: ¿posibles cambios de origen de las importaciones del Paraguay?
}

\author{
Víctor Enciso Cano y Manuela Castillo Quero*
}

Cómo citar: Enciso Cano, V., \& Castillo Quero, M. (2018). Acuerdo UE-MERCosUR: ¿posibles cambios de origen de las importaciones del Paraguay? Ciencia, Economía y Negocios, 2(1), 11-36. doi: http://dx.doi.org/10.22206/ceyn.2018.v2i1.pp11-36

\section{Resumen}

La Unión Europea (UE) y el Mercado Común del Sur (MERCOSUR) han estado negociando la firma de un Acuerdo de Asociación Regional (AAR) desde mediados de la década de los noventa. Los posibles efectos en el MERCOSUR han sido ampliamente estudiados con énfasis en Argentina y Brasil, en detrimento de las dos economías de menor desarrollo relativo: Paraguay y Uruguay; se pretende llenar este vacio focalizando la investigación en Paraguay. El objetivo es identificar los productos importados por Paraguay, a nivel de sub-partidas del Sistema Armonizado de Designación y Codificación de Mercaderías (SA), que podrían ser reemplazados desde los proveedores tradicionales por similares provenientes de la UE, lo que se conoce como desvío de comercio en la literatura especializada. La metodología aplicada se organizó en tres pasos. En el primero, se ajustaron las bases de datos de comercio. Luego, se calcularon los índices de comercio y, finalmente, estos índices se combinaron siguiendo la condicional "si....... entonces...", lo que dio como resultado final la agrupación de las sub-partidas en ocho categorías. Los resultados han mostrado que al menos el 90\% del valor importado por Paraguay, desde sus principales mercados proveedores (China, Brasil y Argentina), pueden ser reemplazados por proveedores similares provenientes desde la UE.

Palabras clave: Unión Europea; MERCOSUR; Paraguay; indicadores de comercio; desvío de comercio.

Clasificación JEL: F13, F14, F15.

\footnotetext{
* Enciso Cano, Víctor: Facultad de Ciencias Agrarias. Universidad Nacional de Asunción. Ruta Mcal. Estigarribia Km 10,5, San Lorenzo-Paraguay venciso@agr.una.py (autor de contacto: venciso@agr.una.py). Castillo Quero, Manuela: Universidad de Córdoba. Dirección: Campus universitario de Rabanales, Edificio Gregor Mendel, $3^{\text {a }}$ Planta, 140014 Córdoba, España (es1caqum@uco.es).
} 


\title{
EU-MERCOSUR Agreement: possible changes in the origin of Paraguay's imports?
}

\author{
Víctor Enciso Cano and Manuela Castillo Quero*
}

\begin{abstract}
The European Union (EU) and the Southern Common Market (MERCOSUR) have been negotiating a bi-regional Association Agreement since the mid-nineties. The possible effects on MERCOSUR have been widely studied with emphasis on Argentina and Brazil, the main economies, and to a lesser extent on the two relatively less developed economies: Paraguay and Uruguay. This work aims to fill this gap by focusing in Paraguay. The objective is to identify products, at the level of sub-headings of the Harmonized System, imported by Paraguay that could be replaced from traditional suppliers by similar ones from the $E U$, called trade diversion in the specialized literature. The applied methodology was organized in three steps. First trade databases were adjusted. Then the trade indices were calculated and finally these indices were combined following the conditional "if ... then ...". The results showed that at least $90 \%$ of the value imported by Paraguay from its main suppliers (China, Brazil and Argentina) could be replaced by similar ones from the $E U$.
\end{abstract}

Keywords: EU; MERCOSUR; Paraguay; trade indicators, trade diversion.

Clasification JEL: F13, F14, F15.

* Enciso Cano, Víctor: Facultad de Ciencias Agrarias. Universidad Nacional de Asunción. Ruta Mcal. Estigarribia Km 10,5, San Lorenzo-Paraguay venciso@agr.una.py (contact author: venciso@agr.una.py). Castillo Quero, Manuela: Universidad de Córdoba. Address: Campus University Campus of Rabanales, Gregor Mendel Bld, $3^{\text {rd }}$ floor, 140014 Córdoba, Spain(es1caqum@uco.es). 


\section{Introducción}

La Unión Europea (UE) y el Mercado Común del Sur (MERCOSUR) están negociando la firma de un Acuerdo de Asociación Regional (AAR) desde mediados de la década de los noventa. En setiembre de 2004, luego de que el intercambio de propuestas de acceso a mercado fuera insatisfactorio para las partes, las negociaciones fueron suspendidas. Años más tarde, en 2010, las negociaciones se retomaron y a la fecha continúan sin haber llegado a un acuerdo. Recientemente, en mayo de 2016, las partes intercambiaron propuestas de acceso a mercado. Los posibles efectos del AAR, tanto en el MERCOSUR como en la UE y los demás países, han sido ampliamente estudiados.

En el MERCOSUR el énfasis ha estado sesgado en medir los impactos en las grandes economías del bloque, Argentina y Brasil, en detrimento de las dos economías de menor desarrollo relativo: Paraguay y Uruguay. Igualmente, los estudios han estado concentrados en evaluar el impacto en las variables macroeconómicas -PIB, exportaciones e importaciones totales- o en sectores específicos - carnes, cereales, manufacturas, etc.- - En tanto que los estudios que se focalizan en productos desagregados (sub-partidas) son escasos. Este trabajo pretende llenar este vacío focalizando la investigación en productos desagregados y en Paraguay. El objetivo de esta investigación es identificar los productos importados por Paraguay, a nivel de sub-partidas del Sistema Armonizado de Designación y Codificación de Mercaderías (SA), que podrían ser reemplazados desde los proveedores tradicionales por proveedores similares provenientes de la UE.

\section{Comercio del Paraguay}

Paraguay se halla ubicado en el centro de Sudamérica, entre los paralelos $19^{\circ} 18^{\prime \prime}$ y $27^{\circ} 30^{\prime \prime}$ y los meridianos $54^{\circ} 19^{\prime \prime}$ y $62^{\circ} 38^{\prime \prime}$ oeste, cubriendo una superficie de poco más de $406.752 \mathrm{~km}^{2}$. Posee una población de 6 millones de habitantes, de los cuales el $61 \%$ es menor de 30 años. La agricultura es el sector más importante en la economía 
paraguaya; conjuntamente con las manufacturas de origen agropecuario, genera el 35\% del PIB, un tercio del empleo y el $77,7 \%$ de las divisas al país (Instituto para la Integración de América Latina y el Caribe, 2016). El Banco Mundial clasifica al Paraguay como un país de ingreso medio-alto. Es la economía de menor desarrollo relativo dentro del MERCOSUR, con un Producto Interior Bruto de 27.623 mil millones de dólares y un ingreso nacional bruto per cápita de 4.161 dólares, datos que corresponden al año 2015. Uno de cada cinco habitantes se encuentra en estado de pobreza, siendo la esperanza de vida igual a 72 años. La tasa de alfabetismo de la población adulta es del 95\%, aproximadamente (Banco Central del Paraguay, 2012).

Hasta la década de los sesenta, el Paraguay era una sociedad agraria, basada en una agricultura atrasada y estancada. En la década de los setenta, la economía del Paraguay vivió años de excepcional crecimiento como producto de la construcción conjunta con el Brasil de la gigantesca represa de Itaipu y debido al incremento en la producción agrícola originado en el cambio en la propiedad de la tierra y la implementación del programa de reforma agraria (Campos, 2010). Sin embargo, desde principios de los años ochenta el Paraguay entró en una senda de bajo o nulo crecimiento económico y no ha sido capaz de mantener un nivel de crecimiento superior a la tasa poblacional sino a partir del año 2008. En ese año los precios de los commodities agroalimentarios experimentaron un alza histórica, con lo cual el Paraguay pudo superar el pico del ingreso per cápita real alcanzado en 1981. Entre 2008 y 2015, el PIB de Paraguay llegó, en un par de ocasiones, a crecer a tasas de dos dígitos inclusive.

Paraguay es un actor pequeño en el comercio mundial, con una participación que no llega al 1\%. Su patrón comercial es similar al de los demás países de Latinoamérica, exporta productos primarios o en los primeros estadios de su procesamiento e importa manufacturas y bienes de capital. El MERCOSUR ha sido históricamente el principal destino de sus exportaciones, con una participación promedio del $30 \%$, aunque en algunos años llegó a superar el 60\%. En los últimos años, el peso relativo del citado mercado en las ventas externas ha disminuido casi linealmente ante el aumento de las exportaciones a 
otros mercados como la UE, Chile y Rusia. Datos de la Organización Mundial de Comercio indican que en 2016 las exportaciones de Paraguay, en términos de valor, se dirigieron a Brasil $(35 \%)$, UE (14\%), Argentina (10\%), Rusia (8\%) y Chile (6\%), entre los principales destinos. Las principales exportaciones de Paraguay se concentran en semillas y frutos oleaginosos (soja principalmente), combustibles (energía eléctrica), carne y despojos comestibles (carne bovina), residuos y desperdicios de las industrias alimentarias (harina de soja), cereales (trigo, maíz y arroz), aceite y grasas (aceite de soja).

Las exportaciones del Paraguay al MERCOSUR se concentran en pocos productos. En la primera mitad de la década de los noventa, el algodón fue la principal exportación del país. Llegó a sumar la mitad del valor exportado al MERCOSUR; luego, por cuestiones fitosanitarias fue dejado de lado, surgiendo en su reemplazo la soja, sus derivados y los cereales. Estos han llegado a sumar prácticamente el $60 \%$ del valor exportado al MERCOSUR. Las importaciones del Paraguay consisten principalmente de bienes de consumo final y bienes de capital. Tres cuartos del valor total importado en los últimos diez años correspondieron a estos productos, más específicamente máquinas y artefactos mecánicos, máquinas y aparatos eléctricos, combustibles y aceites minerales y vehículos automotores. Los principales mercados proveedores en este período han sido China (27\%), Brasil (24\%), Argentina (15\%), la UE (8\%) y Estados Unidos (7\%).

\section{Marco teórico}

\subsection{La integración comercial}

La integración se puede definir como una unión o instancia en la cual los países o las economías se unen con el objetivo de acelerar su tasa de expansión global y racionalizar su crecimiento. Maesso (2011) indica que los primeros antecedentes de la integración se pueden encontrar en la Gran Bretaña del siglo XVIII, y en Alemania e Italia, en el siglo XIX. Sin embargo, el fenómeno integracionista toma impulso tras la Segunda Guerra Mundial, siendo la Comunidad Económica Europea (CEE) el ejemplo más importante. Otras iniciativas tuvieron 
menos éxito por diversos motivos. Según Bhagwati (1992), en parte, fue debido a la reticencia de Estados Unidos en aplicar el artículo XXIV del GATT, dando preferencia a acuerdos multilaterales ante bilaterales. El citado artículo permite los acuerdos regionales, siempre que una parte sustancial del comercio entre las partes del acuerdo sea liberada, pero sin especificar la magnitud de tal liberación. La segunda ola de regionalismo que se inició en los años ochenta tuvo mayor éxito debido, en gran parte, a iniciativas liberadas por los Estados Unidos haciendo uso del artículo XxIv del GATT (Bhagwati, 1992).

Desde entonces, han surgido diversos acuerdos importantes, entre los cuales se puede identificar el Tratado de Libre Comercio de América del Norte, la profundización de la Unión Europea, la conformación de bloques económicos en Asia, como la Asociación de Naciones del Sudeste Asiático (ASEAN) o el Mercado Común del Sur (MERCOSUR). En el presente milenio hay que destacar el Acuerdo Transpacífico de Cooperación Económica, más conocido por sus siglas en inglés TPP, que involucra a economías del Pacífico, entre ellas las de EEUU, Canadá, México, Perú, Malasia, Japón, Australia y Vietnam, y las negociaciones entre la UE y los Estados Unidos, conocidas como Tratado Transatlántico de Comercio e Inversiones (TTIP). A la fecha, la Base de Datos de la Organización Mundial de Comercio (OMC) tiene registrados 277 acuerdos comerciales en implementación.

Una de las justificaciones de la integración económica es la ampliación del mercado. Un mercado pequeño no es atractivo para las inversiones, dado que el retorno financiero no sería rentable con la demanda de una economía pequeña. Por el contrario, un mercado ampliado incrementa la demanda, permite obtener economías de escala y de especialización, al tiempo que aumenta y fortalece el poder de negociación del grupo con relación a la capacidad individual de sus integrantes. Además, la integración fomenta a medio plazo la competencia, la disminución de los costos y la movilidad de los factores de producción y, por tanto, la mejora de la eficiencia. De igual manera, en el largo plazo se observan efectos sobre las variables de crecimiento, tales como cambios en la estructura de producción, difusión de 
tecnología y facilidad para acceder al mercado de capitales (Krugman, Obstfield, \& Melitz 2012).

La integración, según Balassa (1965), se clasifica tradicionalmente en cinco categorías en función del nivel de profundización: (i) zona de libre comercio, (ii) unión aduanera, (iii) mercado común, (iv) unión económica, (v) unión total. La forma más elemental y más utilizada la constituyen el área o la zona de libre comercio, que se forma cuando las partes firmantes del acuerdo deciden eliminar las restricciones al comercio entre ellas, pero manteniendo su independencia en cuanto a sus aranceles con terceros países. Cuando los integrantes de un Área de Libre Comercio deciden establecer un Arancel Externo Común (AEC) se llega a una Unión Aduanera. Se avanza hacia un Mercado Común cuando además de la libre circulación de bienes y el establecimiento de un AEC se acuerda la libre circulación de los factores de producción, personas, capitales y servicios. El MERCOSUR, si bien su nombre incluye el término mercado común, se le considera una unión aduanera imperfecta, debido a que aún no permite la libre circulación de factores, además de que cada país mantiene una lista de excepciones al AEC. Cuando los países integrados en un Mercado Común deciden avanzar hacia la armonización de políticas fiscales y monetarias se llega a lo que se denomina Unión Económica. La actual UE surgida del Tratado de Maastricht es lo más cercano a una Unión Económica que se conozca. Finalmente, y más bien como un objetivo ideal, se incluye la Integración Política, que implica la unificación de todas las políticas, los planes y las legislaciones económicas. Supone la pérdida o cesión de gran parte de la autonomía de las partes hacia un ente supranacional que llevará a tener una sola política, un solo plan y una sola legislación económica para todos los países miembros (Solares, 2010).

\subsection{Los efectos de los acuerdos comerciales}

El análisis económico sobre los efectos de los acuerdos comerciales tiene su inicio en el trabajo de Viner (1950), sobre teoría de las uniones aduaneras. Hasta ese momento, los acuerdos comerciales se analizaban con las mismas metodologías aplicadas a la defensa del 
libre comercio: las ventajas comparativas de Ricardo, o la de dotación de factores de Heckscher-Ohlin, entre las más tradicionales. El trabajo de Jacob Viner identificó dos efectos sobre la producción y los flujos comerciales como consecuencia de la integración. Por un lado, el efecto positivo de la creación de comercio, y por el otro, el efecto negativo de desviación de comercio. Estos efectos son conocidos como estáticos o de corto plazo, para diferenciarlos de los efectos dinámicos o de largo plazo (Krugman et al., 2012).

La creación de comercio tiene lugar cuando una de las partes del acuerdo deja de producir un producto para sustituirlo por otro que es importado del nuevo socio comercial. Se considera que con esto se mejora la asignación de recursos y el bienestar general, al evidenciar las ventajas de la especialización y el comercio de los países que se integran. Es un efecto positivo del acuerdo comercial. Por su lado, en el desvío del comercio una de las partes reemplaza la importación de un producto desde un país que, además de no formar parte del acuerdo, es más eficiente en la producción de este, por otro similar, pero proveniente de una contraparte del acuerdo. Este nuevo proveedor tiene ventajas sobre el anterior debido a los mecanismos de protección o preferenciales acordados en el tratado. Es un efecto negativo del acuerdo comercial.

\subsection{Metodologías de evaluaciones de impacto de los acuerdos co- merciales}

Diversas metodologías han sido utilizadas para evaluar el impacto de los acuerdos comerciales en las economías de las partes involucradas y en los demás países, cada una con sus ventajas y desventajas. Así, se tienen estadísticas descriptivas y modelización, estimaciones econométricas y simulaciones, evaluaciones ex antes y ex post, modelos gravitacionales (United Nations Conference on Trade and Development, 2012; Trejo, 2009). Además, teniendo en cuenta la naturaleza del análisis económico, se utilizan modelos de equilibrio parcial o equilibrio general. Las metodologías antes citadas dan resultados en forma agregada. Kirkpatrick \& George (2009), utilizando un modelo de Equilibrio General Computable EGC, identificó que en un 
escenario de libre comercio UE-MERCOSUR el sector con mayor beneficio para este último, en términos de crecimiento en las exportaciones, serían los "alimentos procesados", carne bovina procesada, aceites vegetales y grasas, productos lácteos, arroz procesado, azúcar, bebidas y productos de tabaco. La desagregación, por ejemplo, de carne y aceites, involucra por lo menos a unas 100 sub-partidas. Este inconveniente unido a la mayor complejidad matemática puede no hacer recomendable el uso de esta metodología dependiendo de los objetivos perseguidos, como los que aquí se plantean, la identificación de productos con elevado nivel de detalle.

Alternativamente a la modelización, cabe el uso de un enfoque más descriptivo mediante el uso de indicadores de comercio exterior y de barreras comerciales-índice de complementariedad, de ventajas comparativas, etc., que es el enfoque que se utiliza en este trabajo y que se describe más adelante. Los indicadores de comercio son útiles para identificar productos que modificarían sus valores de comercio (importación y/o exportación) como consecuencia de la implementación de un acuerdo comercial. No brindan información sobre la magnitud del cambio, ni en el comercio ni en la producción de los bienes.

\section{Metodología}

La presente investigación utilizó indicadores de comercio y se fundamentó en metodologías empleadas en el Centro de Economía Internacional (2003) y en la Asociación Latinoamericana de Integración (2002). Ambas, combinando indicadores de comercio, identificaron productos que podrían ser afectados positiva y negativamente por la firma de un acuerdo comercial con la UE. La primera fue específica para Argentina, en tanto que la segunda abarcó los países de ALADI, excepto México.

En el presente trabajo la identificación de productos que podrían ser objeto de desvío de comercio de parte de Paraguay se realizó a nivel de sub-partidas arancelarias, 6 dígitos del Sistema Armonizado de Designación y Codificación de Mercancías (SA). Los datos de co- 
mercio utilizados correspondieron al período 2010-2012, que fueron los más actualizados al momento de iniciar el estudio. Se prefirió no extender el período a cinco años para evitar trabajar con datos correspondientes a los años de la crisis financiera ocurrida en 2008 y 2009, por considerar que no son representativos del flujo comercial entre las partes involucradas en la investigación. Los datos de comercio fueron extraídos de la base de datos del Banco Mundial conocida como WITS (World Integrated Trade Solutions). Los datos sobre aranceles y otras barreras arancelarias fueron extraídos de la Base de Datos de Aranceles de la Organización Mundial de Comercio (OMC) y del MERCOSUR.

La metodología aplicada se organizó en tres pasos. En el primer paso, se ajustaron las bases de datos de comercio. El ajuste de los datos consistió en la selección o el filtrado de los datos, manteniendo solamente aquellos bienes exportados por la UE que estuvieron sujetos a algún derecho aduanero o alguna restricción no arancelaria para su ingreso al Paraguay. Los demás productos no fueron considerados. Se asumió que, si el producto ya fue exportado por la UE al Paraguay, a pesar de enfrentar barreras comerciales, mayor razón hay para que se incrementen con las ventajas de acceso que resultaren del acuerdo. En caso de no haber sido exportado al Paraguay, la reducción de aranceles o barreras podría incentivar el comercio hacia este mercado. Se trabajó con la media simple de esos años para evitar efectos coyunturales en el flujo comercial (Terra, 2002).

En la segunda parte, se calcularon los tres indicadores: índice de complementariedad comercial UE-Paraguay (ICC), índice de participación de las importaciones desde la UE en las importaciones de Paraguay (IPI) y ventaja comparativa revelada (VCR) de Argentina, Brasil y China, excluyendo a Paraguay en las exportaciones de estos países. Estos países fueron los principales mercados de importación de Paraguay en el período de estudio, cubriendo el $70 \%$ de las importaciones de ese país en el tiempo señalado: China (30\%), Brasil (25\%) y Argentina (15\%). Antes de avanzar a la tercera parte de la metodología, se presenta una breve descripción de los tres indicadores. 


\section{El Índice de Complementariedad Comercial}

El Índice de Complementariedad Comercial (ICC) mide la relación entre la oferta exportadora de un país y la demanda importadora de su contraparte. El índice fue propuesto por Anderson \& Norheim (1993) y se deriva del "índice de ventaja comparativa revelada" desarrollado por Balassa (1965). El ICC UE-Paraguay midió la relación entre la oferta exportadora del primero con el segundo. La probabilidad de que el comercio entre dos partes se concrete depende en gran medida en que la UE venda lo que Paraguay compre y a la inversa; o sea, que exista una complementariedad comercial entre ellos. La fórmula es la siguiente:

$$
I C C_{A B}^{i}=\frac{\frac{X_{A}^{i}}{X_{A}^{T}}}{\frac{M^{i}}{M^{T}}} * \frac{\frac{M_{B}^{i}}{M_{B}^{T}}}{\frac{M^{i}}{M^{T}}}=\left(V C R_{A}^{i}\right) *\left(D C R_{B}^{i}\right)
$$

donde

$I C_{A B}^{i}=$ Índice de complementariedad comercial sectorial del bien “i” entre los países A y B

$X_{A}^{i}=\quad$ Exportaciones del bien "i” del país A

$X_{A}^{T}=\quad$ Exportaciones totales del país A

$M_{B}^{i}=\quad$ Importaciones del bien "i”" del país B

$M_{B}^{T}=\quad$ Importaciones totales del país B

$M^{i}=\quad$ Importaciones mundiales del bien "i", neto de las importaciones del país A

$M^{T}=\quad$ Importaciones mundiales totales

$V C R_{A}^{I}=\quad$ Ventaja Comparativa Revelada del bien "i” del país A $D C R_{B}^{i}=$ Desventaja Comparativa Revelada del bien "i” del país B 
Un ICC mayor a uno es sinónimo de complementariedad entre la especialización exportadora de un país y la especialización importadora del otro, por lo cual el comercio entre las partes debería darse. Un ICC menor a la unidad indica que no existe complementariedad entre las partes. Finalmente, índices próximos a la unidad indican semejanzas en la especialización importadora y exportadora de ambos países. En caso de que uno de los componentes de la formula (VCR o DCR) sea inferior a la unidad y que, a pesar de esto el ICC sea mayor a uno, el componente mayor compensa al menor (Centro de Economía Internacional, 2003). Sin embargo, Terra (2002), optó por no considerar VCR o DCR inferiores a la unidad, aduciendo que de este modo ganaría mayor precisión. Esta posición es adoptada en el presente estudio.

La UE en las importaciones paraguayas

La participación de la UE como proveedor al mercado paraguayo se calculó mediante el índice de participación en las importaciones (IPI). Este es el cociente entre el valor total de las importaciones paraguayas desde la UE y el valor total importado por Paraguay. Aquellos productos que ya son importados bajo el actual arancel externo común tendrán mayor probabilidad de aumentar con las ventajas que el acuerdo otorgará. Igualmente, si un producto tiene una alta participación (por encima del promedio) y, a la vez, enfrenta elevadas barreras de entrada es un indicador de que el mercado paraguayo es importante y atractivo para la UE. Entonces, con las nuevas concesiones del acuerdo se podrá dar el aumento de las importaciones de los productos en cuestión, ya sea por el desvío desde otros destinos hacia el Paraguay como por el aumento de la producción en los países de la UE.

$$
I P I=\frac{V T I P Y}{V T I P y}
$$

IPI = Índice de Participación en las Importaciones

$V T I_{P y}^{U E}=$ Valor total importado por Paraguay desde la Unión Europea

$\mathrm{VTI}_{\mathrm{Py}}=$ Valor total importado por Paraguay 
Ventaja comparativa revelada sin las exportaciones a Paraguay

El tercer indicador fue la ventaja comparativa revelada sin las exportaciones a Paraguay (VCR). La concepción del concepto de ventajas comparativas se remonta a Torrens y Ricardo, quienes buscaban explicar los beneficios resultantes del comercio exterior, pero fue Balassa, en 1965, quien acuñó el término "Ventaja Comparativa Revelada“(VCR) (ALADI, 2002). Balassa (1965) sostenía que las ventajas comparativas entre los países podían ser reveladas por el flujo de comercio, debido a que en el intercambio de bienes se reflejan los costos relativos al igual que las diferencias que existen entre las partes por factores no necesariamente de mercado. Uno de los usos más frecuentes del índice es la comparación entre las ventajas de dos países, identificando los productos en los cuales cada parte tiene ventajas y desventajas, de modo que un país puede conocer en cuáles bienes tiene capacidad para competir en el mercado internacional.

La ventaja comparativa revelada sin las exportaciones a Paraguay (VCR) se calcula para los principales proveedores del Paraguay: China, Brasil y Argentina. Con esto se busca determinar si la competitividad internacional del producto para los proveedores en cuestión se mantiene o es independiente de las ventas hechas al Paraguay. Cuando un producto exportado al mundo por uno de los proveedores tiene VCR mayor a 1 y se mantiene luego de eliminar de sus exportaciones las destinadas a Paraguay, entonces se puede afirmar que ese país -China, Brasil y Argentina- podrá competir con las exportaciones originadas en la UE y no debería ser desplazado del mercado paraguayo. Es decir, el país exporta el producto a precios internacionales, "con lo cual no debería ser desplazado fácilmente ni debería ser un producto donde se genere un aumento de importaciones totales" (Centro de Economía Internacional, 2003).

$$
V C R i=\frac{\frac{X_{A}^{i}}{X_{A}^{t}}}{\frac{X_{W}^{i}}{X_{W}^{t}}}
$$


VCRi: Índice de ventajas comparativas reveladas

$X_{A}^{i}=$ Valor total exportado del producto " $i$ " por el país A (Argentina $^{1}$ ), excluidas las destinadas al Paraguay.

$X_{A}^{t}=\quad$ Valor total exportado por el país "A" (Argentina)

$X_{W}^{i}=$ Valor total exportado globalmente del producto “i”

$X_{M}^{t}=$ Valor total exportado globalmente (mundial)

Finalmente, en la tercera parte se aplicó la matriz del Cuadro 1 para identificar los productos que podrían cambiar desde el proveedor histórico o tradicional hacia la UE, una vez que el acuerdo entre en vigencia. Se combinaron los tres índices horizontalmente siguiendo la condicional "si... entonces...", lo que dio como resultado final la agrupación de las sub-partidas en ocho categorías, donde la categoría I es la que presenta mayor probabilidad de desplazamiento de sus ventas, desde los proveedores tradicionales al Paraguay por importaciones desde la UE.

Los productos agrupados en la categoría I son aquellas sub-partidas: (i) con ICC UE-Paraguay superior a la unidad, indicando coincidencia entre la oferta exportadora del primero con la demanda exportadora del segundo, (ii) con alto nivel de intercambio comercial entre las partes, demostrado por el IPI superior al promedio, iii) en las cuales los proveedores del Paraguay registran una ventaja comparativa revelada, excluidas las exportaciones al Paraguay, igual o inferior a la unidad. En tanto que la menor amenaza la tienen los bienes de la categoría VIII, cuyo ICC es menor o igual a la unidad, demostrando la ausencia de complementariedad entre lo que uno vende y lo que el otro compra; el intercambio comercial entre ambas partes es bajo, o sea IPI menor o igual al promedio, y finalmente la VCR de los tres principales mercados para los bienes de la categoría es superior a la unidad, incluso cuando se excluyen las exportaciones a Paraguay de sus ventas externas totales.

1 Usando a Argentina como ejemplo. 
Cuadro 1: Matriz de Categorización

\begin{tabular}{llll}
\hline ICC & IPI & VCR & Categoría \\
\hline & $>$ promedio & $<=1$ & $\mathrm{I}^{*}$ \\
& & $>1$ & $\mathrm{II}$ \\
& $<=$ promedio & $<=1$ & $\mathrm{III}$ \\
& & $>1$ & $\mathrm{IV}$ \\
& $>$ promedio & $<=1$ & $\mathrm{~V}$ \\
$<=1$ & $>1$ & $\mathrm{VI}$ \\
& & $<=1$ & $\mathrm{VII}$ \\
& $<=$ promedio & $>1$ & $\mathrm{VIII} * *$ \\
\hline
\end{tabular}

Fuente: Adaptado del Centro de Economía Internacional (2003).

"Mayor probabilidad de aumento de las importaciones desde la UE.

${ }^{* *}$ Menor probabilidad de aumento de las importaciones desde la UE.

\section{Resultados y discusión}

\subsection{Características generales de las importaciones paraguayas}

En el período de estudio, Paraguay importó 4.222 sub-partidas por un valor de 11.313 millones de dólares por año, en promedio. Estas consistieron principalmente en bienes de consumo final y bienes de capital, que representaron tres cuartos del valor total importado en el período indicado. En tanto que los bienes intermedios ocuparon el tercer lugar, con un peso de $17,3 \%$. La mayor parte del valor total importado estuvo concentrado en cuatro capítulos del Sistema Armonizado, que sumaron el 59\% del total importado. Estos fueron el capítulo 85 (máquinas, aparatos y artefactos eléctricos), con el 17\%; capítulo 84 (máquinas, aparatos y artefactos mecánicos), con el 14\%; capítulo 27 (aceites y combustibles minerales), con el 14\%; y capítulo 87 (vehículos, automóviles y tractores, con el $9 \%$.

Los principales mercados de importación fueron China (30\%), Brasil (25\%) y Argentina (15\%). Las importaciones desde EE. UU. alcanzaron el 6\% y desde Japón el 3\%. Entre los países europeos, Alemania fue el principal mercado, con una participación del 2\%. La 
UE como un solo mercado tuvo una participación levemente superior a la de Estados Unidos. Por lo tanto, se puede afirmar que los países desarrollados no fueron proveedores importantes del Paraguay durante el período de estudio.

Las importaciones paraguayas desde China sumaron 3.426 millones de dólares por año durante el período de estudio. Los principales capítulos dentro de las importaciones fueron el capítulo 85, con el $41 \%$, seguido del capítulo 84, que representó el 20\%; el capítulo 95 (juguetes y artículos deportivos), con el 14\%; y el capítulo 87, con el $5 \%$. Hay que indicar que muchos de estos productos fueron importados para luego ser re-exportados al mercado brasilero, principalmente como re-exportación no registrada, también conocida como turismo de compra (Campos, 2010). Las importaciones desde Brasil alcanzaron 2.799 millones anuales durante el período de estudio. Los principales capítulos importados (con un valor superior a los 100 millones de dólares) fueron el 84 , con el 15\%; el 31, con el 12\%; el 27, con el 10\%; el 87 , con el $8 \%$, y el 39 (plásticos y sus manufacturas), con el $4 \%$.

Las importaciones desde Argentina alcanzaron 1.739 millones anuales durante el período de estudio. Los principales capítulos importados fueron el 27, con el 39\%; el 72 (hierro y acero), con el $6 \%$, y el 84 , con el 5\%. Luego, se ubicaron los capítulos 38, 39, 87 y 22 (bebidas, líquidos alcohólicos y vinagre), cada uno con una participación del $4 \%$ en el valor total importado desde Argentina. Las importaciones desde Argentina estuvieron menos concentradas que las provenientes de Brasil. Solamente las correspondientes a combustibles minerales superaron los 100 millones de dólares.

En el período analizado, la UE exportó al mercado de Paraguay 3.105 sub-partidas, por un valor de 688,65 millones de dólares. De estas, 910 bienes por un valor de 436,95 millones de dólares tuvieron una participación superior al promedio, indicando que las importaciones desde el mercado comunitario están concentradas en pocos productos. Las manufacturas fueron las principales exportaciones comunitarias al Paraguay, representando el $88 \%$ del valor total exportado a este país. Al mismo tiempo, la UE fue el principal proveedor de productos de varios capítulos. Estos fueron el capítulo 81, metales co- 
munes y sus manufacturas, con el $85 \%$; capítulo 51, lana, con el 52\%; capítulo 97, objetos de arte o colección, con el 42\%; aceites esenciales y perfumería, con el $30 \%$; productos editoriales y de prensa, con el $29 \%$; bebidas, con el $26 \%$, y productos farmacéuticos, con un $24 \%$. Sin embargo, estos capítulos tuvieron un bajo peso relativo en el valor total importado por Paraguay. Todo esto refuerza lo ya expresado: la UE no es un mercado importante para las importaciones del Paraguay, al igual que en las exportaciones comunitarias, el Paraguay tiene baja importancia relativa.

\section{2 Índice de Complementariedad Comercio Unión Europea- Paraguay}

El cálculo del ICC mostró que el $60 \%$ del valor y el $30 \%$ de las sub-partidas correspondieron a productos cuyo ICC UE-Paraguay fue superior a la unidad. De modo que el $40 \%$ restante se realiza basado en otros factores ajenos a la complementariedad comercial. Luego de calculado el ICC y considerando únicamente los productos con índice superior a la unidad, el valor total exportado tuvo una fuerte reducción en los dos capítulos de bienes de capital: el capítulo 87 y el capítulo 84; a pesar de ello, en la estructura de participación no se dieron cambios. Las principales exportaciones de bienes con complementariedad comercial entre la UE y el Paraguay continuaron siendo los capítulos 87 , con el $20 \%$, y el capítulo 84 , con el $16 \%$, seguido de bebidas y líquidos alcohólicos, con el 13\%, y aceites esenciales, perfumería (capítulo 33), con el 10\%. Otros capítulos ubicados entre los más importantes, pero con menor peso relativo fueron el de papel y cartón (capítulo 48), con el 7\%, máquinas y aparatos eléctricos (capítulo 85 ), con el $5 \%$, y productos farmacéuticos (capítulo 30), con el $3 \%$.

\subsection{Ventajas comparativas reveladas}

En el caso de China, luego de calcular la VCR, en la cual se excluyeron los productos destinados al Paraguay, la estructura no presentó ningún cambio. Los mismos capítulos que ocuparon las primeras posiciones, de acuerdo a su valor de importación previo al cálculo de las 
ventajas, se mantuvieron como los más importantes. En el capítulo 87 se dieron las reducciones más importantes y fueron igual al 27\%, mientras que en los capítulos 95, 85 y 84 solamente fueron eliminados unos pocos productos que no representaron más del $5 \%$ del total importado por Paraguay. Entonces, desde la perspectiva de las ventajas comparativas, las importaciones europeas no afectarán a la mayor parte de las ventas de China a Paraguay.

En el caso de los socios del MERCOSUR, Brasil y Argentina, la situación fue muy distinta a la de China. Considerando únicamente las ventajas comparativas excluidas del Paraguay, los dos socios comerciales verán amenazadas la mitad de sus exportaciones al Paraguay por otras provenientes de la UE. No obstante, menos del $2 \%$ de las exportaciones de Brasil y Argentina tienen como destino el Paraguay.

En el caso específico de Brasil, los productos que poseen ventajas comparativas y que fueron importados por el Paraguay sumaron el $51 \%$ de todas las importaciones paraguayas desde el Brasil. Es decir, la mitad de los productos perdieron su ventaja frente a las importaciones de la UE, siendo factibles de ser sustituidas tomando en cuenta únicamente este indicador. El capítulo con la mayor disminución fue el 27, que quedó prácticamente sin ningún valor luego de estimada la VCR que excluía las ventas a Paraguay. El capítulo 31 (fertilizantes) redujo su valor en un $13 \%$, el capítulo 87 en un $36 \%$ y el capítulo 84 en un $40 \%$. Los capítulos 39 (plásticos y sus manufacturas) y el capítulo 85 tuvieron una disminución muy similar, 37 y 36\%, respectivamente.

En cuanto a Argentina, los productos que mantuvieron sus ventajas comparativas reveladas y que fueron importados por el Paraguay sumaron el $48 \%$ de todas las importaciones paraguayas desde Argentina. Este valor fue muy similar al de Brasil, indicando que prácticamente la mitad de la importaciones paraguayas desde el MERCOSUR podrían ser sustituidas por otras provenientes de la UE. Luego de excluir al Paraguay, no se observaron variaciones importantes en el peso relativo de los principales capítulos en el valor total importado desde Argentina. En forma conjunta, los siete capítulos que representaron el $64 \%$ de las importaciones, antes del ajuste en la estimación de la 
VCR de cada producto, mantuvieron el mismo peso relativo luego de eliminar aquellos bienes con índice menor a la unidad, aunque en valores nominales hayan disminuido.

\subsection{Productos con potencial de reemplazo}

El resultado de aplicar la matriz a las importaciones paraguayas desde el mercado chino se presenta en el Cuadro 2. El número de sub-partidas que el Paraguay importó desde China y, a la vez, desde la UE sumaron el $98 \%$ del valor total importado desde el país asiático. Dicho de otro modo, tan solo 346 sub-partidas, igual al 2\% del valor importado desde China, no serían sustituidas por importaciones desde el mercado comunitario, ya que este no es proveedor del mercado paraguayo. Los bienes en las primeras cuatro categorías son las que presentan mayor amenaza de sustitución, debido a que la complementariedad entre la oferta comunitaria y la demanda paraguaya es superior a la unidad. En ellas están la mayor parte del número de sub-partidas y prácticamente la totalidad del valor importado por $\mathrm{Pa}-$ raguay, al igual que más del $90 \%$ de las provenientes desde la UE y desde China.

CUADRO 2: SUb-PARTIDAS CON CRECIMIENTO POTENCIAL DE IMPORTACIONES DE Paraguay desde la UE en detrimento de China

\begin{tabular}{lrrrr}
\hline Categoría & Sub-partidas & $\begin{array}{c}\text { VTI Paraguay } \\
\text { (Miles USD) }\end{array}$ & $\begin{array}{c}\text { VTI Para- } \\
\text { guay-UE (Miles } \\
\text { USD) }\end{array}$ & $\begin{array}{c}\text { VTI Para- } \\
\text { guay-China } \\
\text { (Miles USD) }\end{array}$ \\
\hline Categoría 1 & 180 & 682,290 & 302,410 & 27,887 \\
Categoría 2 & 91 & 120,542 & 46,664 & 30,653 \\
Categoría 3 & 540 & $4,157,223$ & 140,101 & 218,682 \\
Categoría 4 & 629 & $4,432,427$ & 62,548 & $2,880,527$ \\
Categoría 5 & 181 & 97,270 & 29,932 & 7,238 \\
Categoría 6 & 104 & 34,116 & 9,654 & 6,368 \\
Categoría 7 & 365 & 221,482 & 12,749 & 34,134 \\
Categoría 8 & 428 & 298,160 & 10,924 & 157,033 \\
Total & 2,518 & $10,043,510$ & 614,982 & $3,362,521$ \\
\hline
\end{tabular}

Fuente: elaboración propia basada en datos de WITS. 
En el caso de China, la amenaza de sustitución será mayor con relación a las de Argentina y Brasil, ya que continuará sujeta al arancel externo común. Además, deberá enfrentar las importaciones desde la UE, que tendrán un precio relativo inferior con la implementación del acuerdo comercial. Los resultados de la matriz muestran que China quedará prácticamente excluida como proveedora del mercado paraguayo, una vez puesta en vigencia el acuerdo comercial entre el MERCOSUR y la UE.

Tomando en consideración los principales productos dentro de cada una de las primeras cuatro categorías, que fueron las más amenazadas de ser sustituidas por productos provenientes de la UE, según la matriz aplicada, se observó una alta concentración del valor en unos pocos productos. Por lo general, los dos bienes de mayor valor tuvieron una participación en el valor total importado superior o igual a dos dígitos, mientras que los demás valores relativos fueron inferiores al 10\%. Esto implica que sustituir el origen de las importaciones de unos pocos bienes tendrá un importante efecto sobre la estructura de las importaciones. La mayoría de bienes amenazados en las importaciones provenientes de China fueron aparatos y artefactos eléctricos y mecánicos, vehículos automotores y sus partes y productos relacionados con grabación y reproducción de sonidos. Estos fueron además los capítulos con mayor peso en las importaciones paraguayas desde China. Dicho de otra manera, la amenaza de sustitución se daría en las principales sub-partidas importadas por Paraguay desde China.

El resultado de aplicar la matriz a las importaciones paraguayas desde el mercado brasilero se presenta en el Cuadro 3. El número de sub-partidas que el Paraguay importó desde Brasil y, a la vez, desde la UE sumó el 98\% del valor total importado desde el país vecino. Es decir, solamente 530 sub-partidas, que sumó el $2 \%$ del valor importado por Paraguay desde Brasil, correspondieron a productos que la UE no exportó al Paraguay. En las primeras cuatro categorías se concentró más de la mitad del número de sub-partidas amenazadas y, al mismo tiempo, la mayor parte, en términos de valor, de las importaciones totales del Paraguay para los productos de las ocho categorías, 
así como al menos el $90 \%$ del valor total importado tanto desde la UE como desde Brasil.

Por lo antes planteado, si en las condiciones pre-acuerdo fueron importadas por Paraguay, una vez que los bienes comunitarios tengan el mismo trato arancelario que Brasil, las probabilidades de que las compras desde la UE se incrementen son mayores. Concluyendo, los resultados mostraron que las importaciones paraguayas desde el Brasil serían fuertemente amenazadas por parte de importaciones similares provenientes desde la UE cuando se liberalice el intercambio comercial entre las partes.

CuAdro 3: Sub-PARTIDAS CON CRECIMIENTO POTENCIAL DE IMPORTACIONES DE Paraguay desde la UE en detrimento de Brasil

\begin{tabular}{lrrrr}
\hline Categoría & Sub-partidas & $\begin{array}{c}\text { VTI Paraguay } \\
\text { (Miles USD) }\end{array}$ & $\begin{array}{c}\text { VTI Para- } \\
\text { guay-UE (Miles } \\
\text { USD) }\end{array}$ & $\begin{array}{c}\text { VTI Para- } \\
\text { guay-Brasil } \\
\text { (Miles USD) }\end{array}$ \\
\hline Categoría 1 & 238 & 669,401 & 252,048 & 88,024 \\
Categoría 2 & 33 & 162,042 & 84,441 & 36,791 \\
Categoría 3 & 1,027 & $6,570,940$ & 148,620 & $1,120,087$ \\
Categoría 4 & 257 & $2,318,872$ & 61,039 & $1,320,081$ \\
Categoría 5 & 271 & 127,181 & 38,448 & 21,324 \\
Categoría 6 & 15 & 7,102 & 1,766 & 2,697 \\
Categoría 7 & 801 & 435,412 & 25,176 & 112,344 \\
Categoría 8 & 105 & 88,427 & 1,201 & 52,715 \\
Total Categorías & 2,747 & $10,379,378$ & 612,739 & $2,754,063$ \\
\hline
\end{tabular}

Fuente: elaboración propia basada en datos de WITS.

Analizando los principales productos dentro de cada una de las primeras cuatro categorías, que fueron las más amenazadas de ser sustituidas con productos provenientes de la UE según la matriz aplicada, se notó que las principales importaciones desde Brasil no tuvieron un peso relativo muy elevado dentro de cada categoría. Con la excepción de la categoría 4, en las demás los productos de mayor peso relativo no sobrepasaron el $25 \%$ del valor total de la categoría. Consecuentemente, el efecto sustitución se distribuiría entre un mayor número de 
productos, lo que llevaría a que el efecto en las importaciones desde ese mercado sea menos acentuado. Las principales sub-partidas con el potencial de ser sustituidas fueron fertilizantes, maquinarias eléctricas y mecánicas, y automotores. Estos productos formaron (y siguen formando) parte de las principales importaciones que el Paraguay realiza desde Brasil.

El resultado de aplicar la matriz a las importaciones paraguayas desde el mercado argentino se presenta en el Cuadro 4. El número de sub-partidas que el Paraguay importó desde Argentina y, a la vez, desde la UE fue 1,938 y sumaron el $98 \%$ del valor total importado desde el país vecino. Es decir, solamente 382 sub-partidas, o el $2 \%$ del valor importado por Paraguay desde Argentina, correspondieron a productos que la UE no exportó al Paraguay. En las primeras cuatro categorías se concentró más de la mitad del número de sub-partidas amenazadas y, al mismo tiempo, la mayor parte, en términos de valor, de las importaciones totales del Paraguay para los productos de las ocho categorías, así como al menos el 90\% del valor total importado tanto desde la UE como desde Argentina. En resumidas cuentas, prácticamente todas las importaciones desde Argentina podrían ser sustituidas con importaciones similares provenientes desde la UE.

Si analizamos los principales productos dentro de cada una de las primeras cuatro categorías, contrario a lo que pueda ocurrir con las importaciones desde Brasil, los efectos de reemplazo se concentraron en un menor número de sub-partidas. Los productos amenazados y con una importante participación en las compras paraguayas desde Argentina fueron el cemento Portland para construcción, los vehículos automotores y los medicamentos. Entre los bienes agroalimentarios, se destacaron los duraznos y las nectarinas. 
CUAdRo 4: Sub-PARTIDAS CON CRECIMIENTO POTENCIAL DE IMPORTACIONES DE Paraguay desde la UE en detrimento de Argentina

\begin{tabular}{lrrrr}
\hline Categoría & Sub-partidas & \multicolumn{1}{c}{$\begin{array}{c}\text { VTI Paraguay } \\
\text { (Miles USD) }\end{array}$} & $\begin{array}{r}\text { VTI Para- } \\
\text { guay-UE } \\
\text { (Miles USD) }\end{array}$ & $\begin{array}{r}\text { VTI Para- } \\
\text { guay-Argentina } \\
\text { (Miles USD) }\end{array}$ \\
\hline Categoría 1 & 222 & 745,566 & 324,130 & 72,281 \\
Categoría 2 & 24 & 89,971 & 27,481 & 8,382 \\
Categoría 3 & 897 & $6,576,104$ & 135,154 & 762,176 \\
Categoría 4 & 230 & $2,322,342$ & 68,725 & 792,250 \\
Categoría 5 & 217 & 129,047 & 37,966 & 6,625 \\
Categoría 6 & 10 & 482 & 135 & 145 \\
Categoría 7 & 652 & 423,198 & 19,313 & 36,206 \\
Categoría 8 & 69 & 100,682 & 5,004 & 34,542 \\
Total Categorías & 2,321 & $10,387,392$ & 617,908 & $1,712,606$ \\
\hline
\end{tabular}

Fuente: elaboración propia basada en datos de WITS.

Para finalizar, un total de 1,111 productos, por un valor de importación de 241,5 millones, igual al 2\% del VTI por Paraguay en el período de estudio, no fueron proveídos por al menos uno de los tres países estudiados. De modo que el $98 \%$ del valor importado estuvo amenazado con ser sustituido, en mayor o menor grado, por productos similares provenientes de la UE. Esto, antes que una amenaza a las exportaciones de los tres países debería ser considerado como una ventaja para el Paraguay, debido a la ampliación o diversificación de la oferta para este país.

\section{Conclusiones}

Los resultados han mostrado que al menos el 90\% del valor importado por Paraguay desde sus principales mercados proveedores China, Brasil y Argentina- puede ser reemplazado por importaciones similares provenientes desde la UE. Esto no es de sorprender al saber que las principales importaciones del país fueron bienes de capital y de consumo final, y que la UE es uno de los principales productores 
de estos tipos de bienes. La competencia que el nuevo socio comercial representará para los tres países como proveedores del mercado paraguayo probablemente no sea de preocupación para estos, debido a la baja participación de Paraguay en sus respectivas exportaciones. Más bien la situación se convierte en una ventaja para el Paraguay ante la ampliación de la oferta de productos, lo que constituye un aspecto positivo.

Un aspecto negativo es el efecto que tendrá el acuerdo comercial sobre el comercio de re-exportación desde Paraguay a Brasil, principalmente de productos importados desde China. El arancel externo común del MERCOSUR hace inviable la importación de tales bienes -por ejemplo, productos del capítulo 95- por parte tanto de Brasil como de Argentina, mientras que Paraguay, por incluirlos en su lista de excepción, los puede importar libremente; luego, son introducidos a Brasil y Argentina por el mecanismo ya explicado anteriormente. Con la inclusión de la UE como socio comercial del acuerdo, los consumidores tanto de Brasil como de Argentina tendrán acceso a similares productos, pero de una calidad superior a los "importados" vía Paraguay. Todo esto llevará a la desaparición de la ventaja que Paraguay posee.

Consecuentemente, desde el punto de vista de las importaciones se tendrán dos efectos contrapuestos. Por un lado, se ampliará la oferta de productos para el Paraguay, que hasta ahora en muchos casos está limitada debido a que el arancel externo común hace inviable su importación desde la UE. Por otro lado, el negocio de re-exportación desde China a los países vecinos finalizará con el consecuente efecto negativo en los ingresos aduaneros y en el empleo. Si bien desde el punto de vista ético y moral esta forma de comercio por parte de Paraguay es cuestionable (muy cuestionable), tendrá efectos negativos en la economía del país. Solicitar acciones defensivas para paliar los efectos negativos posiblemente no tenga una respuesta favorable de los socios. Más bien, Paraguay debería solicitar apoyo desde el componente cooperación del acuerdo para reconvertir la región del país donde se desarrolla esta actividad comercial. 


\section{Referencias}

Anderson K. \& H. Norheim. (1993). From Imperial to Regional Trade Preferences: Its Effect on Europe's Intra and Extra-Regional Trade. Weltwirtschaftliches Archiv, 129(1), 78-102. Recuperado de https://doi.org/10.1007/BF02707488

Asociación Latinoamericana de Integración. (2002). Probable impacto que tendrían los acuerdos con la Unión Europea en el comercio intrarregional y en el comercio de los países miembros con los países de Europa. Recuperado de http://www.aladi.org/ nsfaladi/estudios.nsf/vpublianterioresweb/81B9744B7662B12003256C2A005BD908

Balassa, B. (1965). Trade liberalization and revealed comparative advantages. England: Manchester School, 33(2), 99-123. Recuperado de https://doi.org/10.1111/j.1467-9957.1965.tb00050.x

Banco Central del Paraguay. (2012). Informe Económico Preliminar-Diciembre 2012. Recuperado de https://www.bcp.gov.py/ userfiles/files/attachments/article/149/Informe_Economico_ Preliminar_2012_qreemplaza_030113.pdf

Bhagwati, J. (1992). Regionalism versus multilateralism. The World Economy, 15(5), 535-555. https://doi.org/10.1111/j.1467 -9701.1992.tb00536.x

Campos, L. (2010). Apuntes de historia económica del Paraguay: desarrollo, auge y decadencia de una economía de enclaves. Asunción: Intercontinental.

Centro de Economía Internacional. (2003). Oportunidades y amenazas para la Argentina de un acuerdo MERCOSUR-Unión Europea: un estudio de impacto sectorial. Revista del Centro de Economía Internacional. Secretaría de Comercio y Relaciones Económicas Internacionales. Ministerio de Relaciones Exteriores, Comercio Internacional y Culto. Serie Estudios del CEI. Nro. 3., febrero 2003. Buenos Aires: CEI.

Instituto para la Integración de América Latina y el Caribe. (2016). Informe MERCOSUR Número 21 2015-2016. Serie: Informes Subregionales de Integración. Recuperado de https://publica- 
tions.iadb.org/bitstream/handle/11319/7959/Informe-MERCOSUR-No-21-2015-2016-Segundo-semestre-2015-Primer-semestre-2016.pdf

Kirkpatrick, C. \& George, C. (2009). Trade sustainability impact assessment (SIA) of the association agreement under Negotiation between the European Community and MERCOSUR. Final overview. Final report. Revised March 2009. Recuperado de http://www.sia-trade.org/mercosur/phase2/Mercosur_final_ overview_310309.pdf

Krugman, P., Obstfeld, M., \& Melitz, M. J. (2012). Economía internacional: Teoría y política. Madrid: Pearson.

Maesso, M (2011). La integración económica. Revista Boletín Económico de ICE, 858, 119-132. Recuperado de https://dialnet. unirioja.es $/$ servlet $/$ revista?codigo $=677$

Solares, A. (2010). Integración. Teoría y procesos. Bolivia y la integración. Recuperado de www.eumed.net/libros/2010e/814/

Terra, M (2002). Uruguay y el MERCOSUR frente a un acuerdo con la Unión Europea. Prioridades para la negociación arancelaria. Recuperado de http://www.cepal.org/es/publicaciones/28669-uruguay-y-el-mercosur-frente-un-acuerdo-con-la-union-europea-prioridades-para-la

United Nations Conference on Trade and Development. (2012). A practical guide to trade policy analysis. Geneva: UNCTAD.

Viner, J. (1950). The Custom Union Issue. New York: Carnegie Endowment for International Peace. 